\title{
A Finite Element Method for Calculating the Pressure-drag Flow of Polymers Based on Computational Fluid Dynamics
}

\author{
Chao WANG ${ }^{\mathrm{a},{ }^{*}}$ \\ ${ }^{1}$ College of Computer Science, South-central University for nationalities, Hubei Wuhan, 430074, \\ China \\ a, *wangchaofly@126.com
}

Keywords: Navier-Stock equations, Newtonian fluid model, Pressure-drag flow, FEM.

\begin{abstract}
In this research, a Navier-Stokes equations based Newtonian fluid model was employed to conduct mathematical modeling on the polymer fluids with low shear rate under pressure-drag flow. Then, a weight function for the equations of the continuity and motion of the fluids is obtained by using an interpolation function. Besides, the authors constructed a general FEM equation based on a rheological model by setting the data, and the coordinates of grid nodes, as well as relevant boundary conditions. The obtained values revealed that the flow rate under compound conditions precisely agrees with that under the effect of two single boundary conditions respectively; while the pressure distribution accords well with the set boundary conditions. These have proven the correctness of the solution using the method proposed. The method of changing the ways of the division and the setting of grids can be applied in the FEM analysis of other two dimensional Newtonian rheological models with regular shapes.
\end{abstract}

\section{Introduction}

This work took the pressure drag for the Newtonian fluids of polymer slabs as a research object. Then, Navier-Stock equations were used to solve the flow rate and pressure of the fluids based on FEM [1,2]. The two dimensional rheological models concerning the fluids of the slabs were conducted FEM analysis [3,4]. The values obtained show the distribution of the flow rate and the pressure for the polymer fluids based on the Newtonian rheological model [5]. In addition, the flow characteristics of the polymer fluids under the compound effect of the multiple boundary conditions were compared.

\section{Case Mode and Mathematical Equations}

\section{Geometric Model}

We took the slow steady flow of the fluids for LLDPE material inside the space of the slab as an example, which was used as a Newtonian fluid at low shear rate. The rate of the surface drag within the slab is $u=0.02 \mathrm{~m} / \mathrm{s}$, entrance pressure is $\mathrm{p}=2,000 \mathrm{~Pa}$, and outlet pressure is $\mathrm{p}=0$. The inferior wall (slab) is fixed. Meanwhile, $\mu=1300 \mathrm{~Pa} \cdot \mathrm{s}$ denotes the fluid viscosity at $230^{\circ} \mathrm{C}$. Figure 1 illustrates the relevant simplified model concerning two-dimensional flow field distribution under the compound effect of pressure drag. 


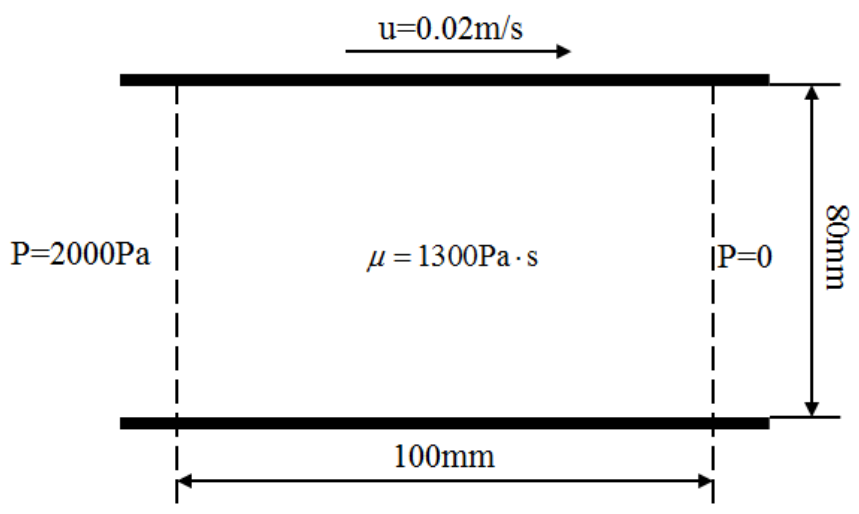

Fig.1 Two-dimensional Newtonian rheological model for the fluids within the slab

\section{Mathematical equations}

Based on the law of conservation of momentum, the relationship between the force applied on the fluids and fluids' motion can be established. The motion equation of aforementioned fluid model can be described using Navier-Stock equations as[6]:

the continuity equation:

$$
\frac{\partial u}{\partial x}+\frac{\partial v}{\partial y}=0
$$

the motion equation along $\mathrm{x}$ direction:

$-\frac{\partial p}{\partial x}+\left(\frac{\partial \tau_{x x}}{\partial x}+\frac{\partial \tau_{x y}}{\partial y}\right)=0$

the motion equation along y direction:

$-\frac{\partial p}{\partial y}+\left(\frac{\partial \tau_{y x}}{\partial x}+\frac{\partial \tau_{y y}}{\partial y}\right)=0$

where, shear stress $\tau$ satisfies the constitutive Newtonian equation as

$\tau=\dot{\mu}=\left[\begin{array}{cc}\tau_{x x} & \tau_{x y} \\ \tau_{y x} & \tau_{y y}\end{array}\right]=\mu\left[\begin{array}{cc}2 \frac{\partial u}{\partial x} & \frac{\partial u}{\partial y}+\frac{\partial v}{\partial x} \\ \frac{\partial v}{\partial x}+\frac{\partial u}{\partial y} & 2 \frac{\partial v}{\partial y}\end{array}\right]$

$\tau_{x x}, \tau_{x y}, \tau_{y x}$ and $\tau_{y y}$ represent the normal stresses, and shear stresses along the $\mathrm{x}$ and $\mathrm{y}$ directions respectively.

\section{Finite Element Solution and Numerical Calculation}

\section{The Grid Division of the Computing Region}

Quadrilateral elements [7] were used to divide the grids in whole computing region, as shown in Figure 2: the whole quadrilateral element is divided into 12 quadrilateral units and then each quadrilateral is subdivided into four small regions according to its middle point. The units corresponding to flow rate are set to be one order higher than that of pressure units. The number of the discrete elements in whole grid is 12 , the total node numbers of flow rate units, and pressure units are 63 and 20 respectively. 


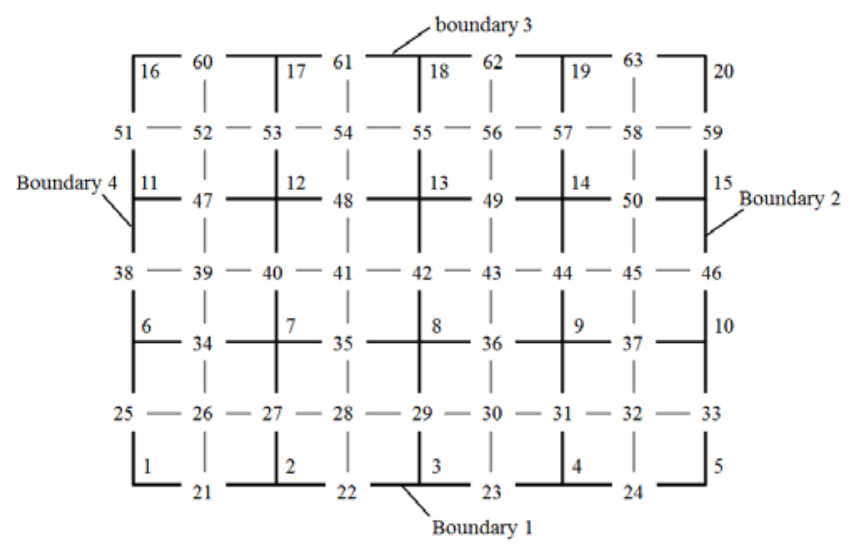

Fig.2 The discrete grids in computing region

The parameters tables obtained based on discretization of grids consist of JMV-the node datum in flow rate unit, JMP-the node datum in pressure unit, JXYV-the coordinate data for the node in flow rate unit, JXYP-the coordinate data for the node in pressure unit, JBV-the boundary conditions of flow rate, and JBP- the boundary conditions of pressure. JMV and JMP are used to store the node number data relating to flow rate, and pressure, units respectively, while JXYV and JXYP can store the coordinate data for the flow rates and pressures in each node. JBV stores the data of boundary node numbers and the flow rates along the $\mathrm{x}$ and $\mathrm{y}$ directions. In addition, JBP is used to store the data including the unit number, the serial number of the edges of the boundary units, the cosine of outward normal at the edge of the boundary- $\cos \theta_{x}$ and $\cos \theta_{y}$, and the pressure values at the first and second points in the edges of boundary.

\section{The Establishment of the Interpolation Function and Unit Equation}

The data of discrete elements were processed using an interpolation function. $\boldsymbol{\Phi}$ and $\psi$ are the interpolation functions for units of flow rate and pressure, separately. The derivatives of the interpolation function $\boldsymbol{\Phi}$ to $\mathrm{x}$ and $\mathrm{y}$ and to the dimensionless coordinates $\xi$ and $\eta$ of the interpolation function present the following relation, where $\boldsymbol{J}$ is the Jacobian matrix.

$$
\left[\begin{array}{l}
\frac{\partial \Phi}{\partial x} \\
\frac{\partial \Phi}{\partial y}
\end{array}\right]=\boldsymbol{J}^{-1}\left[\begin{array}{l}
\frac{\partial \Phi}{\partial \xi} \\
\frac{\partial \Phi}{\partial \eta}
\end{array}\right] \quad \boldsymbol{J}=\left[\begin{array}{ll}
\frac{\partial x}{\partial \xi} & \frac{\partial y}{\partial \xi} \\
\frac{\partial x}{\partial \eta} & \frac{\partial y}{\partial \eta}
\end{array}\right]
$$

By multiplying the interpolation weight function $\psi$ by the continuity equation, a weighted residual equation for the continuity equation is established. Then, this equation is converted into the units of divided grids.

$$
\iint_{\Omega^{e}} \psi\left(\frac{\partial u}{\partial x}+\frac{\partial v}{\partial y}\right) d s d y=0
$$

The flow rate and pressure at each point are expanded using an interpolation function and then substituted into the above formula. Then, we acquire:

$$
\left[\iint_{\Omega^{e}} \boldsymbol{\psi}\left(\frac{\partial \boldsymbol{\Phi}^{T}}{\partial x}\right) \mathrm{dxdy}\right] \boldsymbol{u}_{I}^{e}+\left[\iint_{\Omega^{e}} \boldsymbol{\psi}\left(\frac{\partial \boldsymbol{\Phi}^{T}}{\partial y}\right) \mathrm{dxdy}\right] \boldsymbol{v}_{I}^{e}=0
$$

Where, $\boldsymbol{u}_{I}^{e}$ and $\boldsymbol{v}_{I}^{e}$ denote the vectors composed of the flow rate of each node in units along $\mathrm{x}$ and $\mathrm{y}$ direction, respectively; $\boldsymbol{p}_{I}^{e}$ stands for the vector constituted by pressure of each node in units.

The above formula can be simplified as follows. 


$$
\begin{aligned}
& B_{1}^{e} \boldsymbol{u}_{I}^{e}+B_{2}^{e} \boldsymbol{v}_{I}^{e}=0 \\
& B_{1}^{e}=\iint_{\Omega^{e}} \psi\left(\frac{\partial \boldsymbol{\Phi}^{T}}{\partial x}\right) \mathrm{dxdy}=\int_{-1}^{1} \int_{-1}^{1}\left[\psi\left(\frac{\partial \boldsymbol{\Phi}}{\partial x}\right)\right]|\boldsymbol{J}| d \xi d \eta \\
& B_{2}^{e}=\iint_{\Omega^{e}} \psi\left(\frac{\partial \boldsymbol{\Phi}^{T}}{\partial y}\right) \mathrm{dxdy}=\int_{-1}^{1} \int_{-1}^{1}\left[\psi\left(\frac{\partial \boldsymbol{\Phi}}{\partial y}\right)\right]|\boldsymbol{J}| d \xi d \eta
\end{aligned}
$$

According to the transformational relation between interpolation functions and Jacobian matrices, $\psi, \boldsymbol{J}, \frac{\partial \Phi}{\partial x}$ and $\frac{\partial \Phi}{\partial y}$ are all functions relating to $\xi$ and $\eta$. Therefore, Gauss integral is utilized as follows.

$$
\begin{aligned}
& B_{1}^{e}=\sum_{i=1}^{6} \sum_{j=1}^{6} H_{i} H_{j}\left[\boldsymbol{\psi}\left(\frac{\partial \boldsymbol{\Phi}}{\partial x}\right)\right]|\boldsymbol{J}| \\
& B_{2}^{e}=\sum_{i=1}^{6} \sum_{j=1}^{6} H_{i} H_{j}\left[\boldsymbol{\psi}\left(\frac{\partial \boldsymbol{\Phi}}{\partial y}\right)\right]|\boldsymbol{J}|
\end{aligned}
$$

By substituting Formula (4) into Formula (3) and (2), then being multiplied by the weight function $\Phi$, integration is performed in the computing region. Then, by expanding each item, an interpolation function is introduced and then converted to the units, thus obtaining the following formulas.

$$
\begin{aligned}
& {\left[2 \mu \iint_{\Omega^{e}} \frac{\partial \boldsymbol{\Phi}}{\partial x} \frac{\partial \boldsymbol{\Phi}^{T}}{\partial x} d x d y+\mu \iint_{\Omega^{e}} \frac{\partial \boldsymbol{\Phi}}{\partial y} \frac{\partial \boldsymbol{\Phi}^{T}}{\partial y} d x d y\right] \boldsymbol{u}_{I}^{e}+\left[\mu \iint_{\Omega^{e}} \frac{\partial \boldsymbol{\Phi}}{\partial x} \frac{\partial \boldsymbol{\Phi}^{T}}{\partial y} d x d y\right] \boldsymbol{v}_{I}^{e}} \\
& -\left[\iint_{\Omega^{e}}\left(\frac{\partial \boldsymbol{\Phi}}{\partial x} \boldsymbol{\psi}^{T}\right) d x d y\right] \boldsymbol{p}_{I}^{e}=-\int_{\Gamma^{e}}\left(\boldsymbol{\Phi} \boldsymbol{\psi}^{T} \boldsymbol{p}_{I}^{e}\right) \cos \theta_{x} d \Gamma \\
& {\left[2 \mu \iint_{\Omega^{e}} \frac{\partial \boldsymbol{\Phi}}{\partial y} \frac{\partial \boldsymbol{\Phi}^{T}}{\partial y} d x d y+\mu \iint_{\Omega^{e}} \frac{\partial \boldsymbol{\Phi}}{\partial x} \frac{\partial \boldsymbol{\Phi}^{T}}{\partial x} d x d y\right] \boldsymbol{v}_{I}^{e}+\left[\mu \iint_{\Omega^{e}} \frac{\partial \boldsymbol{\Phi}}{\partial y} \frac{\partial \boldsymbol{\Phi}^{T}}{\partial x} d x d y\right] \boldsymbol{u}_{I}^{e}} \\
& -\left[\iint_{\Omega^{e}}\left(\frac{\partial \boldsymbol{\Phi}}{\partial y} \boldsymbol{\psi}^{T}\right) d x d y\right] \boldsymbol{p}_{I}^{e}=-\int_{\Gamma^{e}}\left(\boldsymbol{\Phi} \boldsymbol{\psi}^{T} \boldsymbol{p}_{I}^{e}\right) \cos \theta_{y} d \Gamma
\end{aligned}
$$

The above two formulas can be simplified as follows.

$$
\begin{aligned}
& D_{11}^{e} \boldsymbol{u}_{I}^{e}+D_{12}^{e} \boldsymbol{v}_{I}^{e}-C_{1}^{e} \boldsymbol{p}_{I}^{e}=-F_{1}^{e} \\
& D_{21}^{e} \boldsymbol{u}_{I}^{e}+D_{22}^{e} \boldsymbol{v}_{I}^{e}-C_{2}^{e} \boldsymbol{p}_{I}^{e}=-F_{2}^{e}
\end{aligned}
$$

Where, $D_{11}^{e}, D_{12}^{e}, C_{1}^{e}, D_{21}^{e}, D_{22}^{e}$ and $C_{2}^{e}$ correspond to the items in the left of Formulas (13) and (14), respectively; while $F_{1}^{e}$ and $F_{2}^{e}$ accord with the items in the right of the two formulas separately. Based on the Gauss integral, these items can be converted as follows using numerical integration. 


$$
\begin{aligned}
& F_{2}^{e}=\sum_{i=1}^{6} H_{i}\left[\left(\boldsymbol{\Phi}^{T} \boldsymbol{p}_{I}^{e}\right) \cos \theta_{y}\right]|\boldsymbol{J}| \\
& D_{12}^{e}=\mu \sum_{i=1}^{6} \sum_{j=1}^{6} H_{i} H_{j}\left(\frac{\partial \boldsymbol{\Phi}}{\partial x} \frac{\partial \boldsymbol{\Phi}^{T}}{\partial y}\right)|\boldsymbol{J}| \\
& D_{21}^{e}=\mu \sum_{i=1}^{6} \sum_{j=1}^{6} H_{i} H_{j}\left(\frac{\partial \boldsymbol{\Phi}}{\partial y} \frac{\partial \boldsymbol{\Phi}^{T}}{\partial x}\right)|\boldsymbol{J}| \\
& D_{22}^{e}=\mu \sum_{i=1}^{6} \sum_{j=1}^{6} H_{i} H_{j}\left(2 \frac{\partial \boldsymbol{\Phi}}{\partial y} \frac{\partial \boldsymbol{\Phi}^{T}}{\partial y}+\frac{\partial \boldsymbol{\Phi}}{\partial x} \frac{\partial \boldsymbol{\Phi}^{T}}{\partial x}\right)|\boldsymbol{J}| \\
& C_{1}^{e}=\sum_{i=1}^{6} \sum_{j=1}^{6} H_{i} H_{j}\left(\frac{\partial \boldsymbol{\Phi}}{\partial x} \boldsymbol{\psi}^{T}\right)|\boldsymbol{J}| \\
& C_{2}^{e}=\sum_{i=1}^{6} \sum_{j=1}^{6} H_{i} H_{j}\left(\frac{\partial \boldsymbol{\Phi}}{\partial y} \boldsymbol{\psi}^{T}\right)|\boldsymbol{J}| \\
& F_{1}^{e}=\sum_{i=1}^{6} H_{i}\left[\left(\boldsymbol{\Phi}_{\boldsymbol{\psi}^{T}} \boldsymbol{p}_{I}^{e}\right) \cos \theta_{x}\right]|\boldsymbol{J}| \\
& F_{2}^{e}=\sum_{i=1}^{6} H_{i}\left[\left(\boldsymbol{\Phi}_{\boldsymbol{\psi}^{T}} \boldsymbol{p}_{I}^{e}\right) \cos \theta_{y}\right]|\boldsymbol{J}|
\end{aligned}
$$

By combining the above formulas, the general equation for the units of discretized grids is obtained. Then, a general equation is obtained through iterative accumulation, where $\mathrm{B}, \mathrm{C}, \mathrm{D}$ and $\mathrm{F}$ are constituted by corresponding element matrices.

$$
\left[\begin{array}{ccc}
D_{11} & D_{12} & -C_{1} \\
D_{21} & D_{22} & -C_{2} \\
B_{1} & B_{2} & 0
\end{array}\right]\left[\begin{array}{c}
u_{I} \\
v_{I} \\
p_{I}
\end{array}\right]=\left[\begin{array}{c}
-F_{1} \\
-F_{2} \\
0
\end{array}\right]
$$

\section{Results of Post-processing}

\section{Calculation Flow}

The whole numerical calculation based on FEM is performed as follows: to begin with, the data of grids including the total number of grid units and nodes, nodes at each boundary and boundary units are read. In addition, the finite element data of flow rate units, JXYV, JMP and JXYP are also read. After setting the viscosity of materials, matrices of boundary conditions for flow rate and pressure are established according to actual situations. Afterwards, all the subblocks of coefficient matrices of units needed by the general equation are calculated so as to constitute the general equation. Finally, the diagonal normalization method is used to solve the general equation to further carry out post-processing of the data obtained.

\section{Data Post-processing}

After obtaining the solutions of the general equation, data of nodes including the coordinates, the flow rates along $\mathrm{x}$ and $\mathrm{y}$ directions and pressure are post-processed by Tecplot software in unstructured data form, as illustrated in Figure 3. Figures $3 a$, $3 b$ and $3 c$ demonstrate the distributions of flow rates under compound boundary conditions, the single effect of drag of the interior wall and the single effect of entrance pressure, respectively. Table 1 displays the comparison 
of the flow rates of outlet nodes under the superimposed effect of single boundary conditions and compound boundary conditions. Based on the data obtained, it can be known that the accumulated results of data obtained under the single drag effect of the interior wall and those acquired under the single effect of entrance pressure equal to the flow rate under the compound effect of these two kinds of boundary conditions. The properties of fluids satisfy the accumulation characteristics of Newtonian fluids. In addition, it can be found from Table 1 that the pressure of fluids within the slab gradually reduces from the entrance, while numerical values of the contour of pressure in the direction perpendicular to the slab remain the same, corresponding to the set boundary conditions.

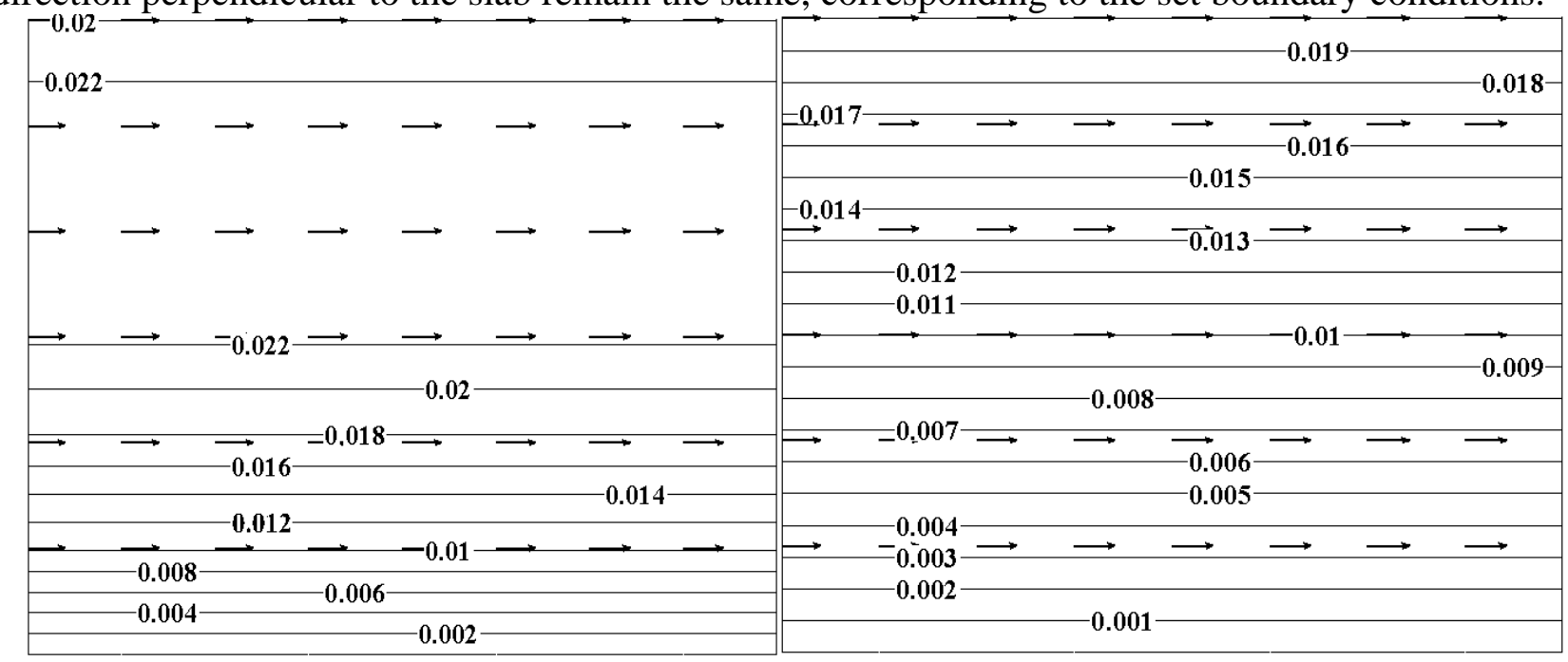

a) distribution of flow rate under compound boundary conditions; b) distribution of flow rate under the drag of interior wall;

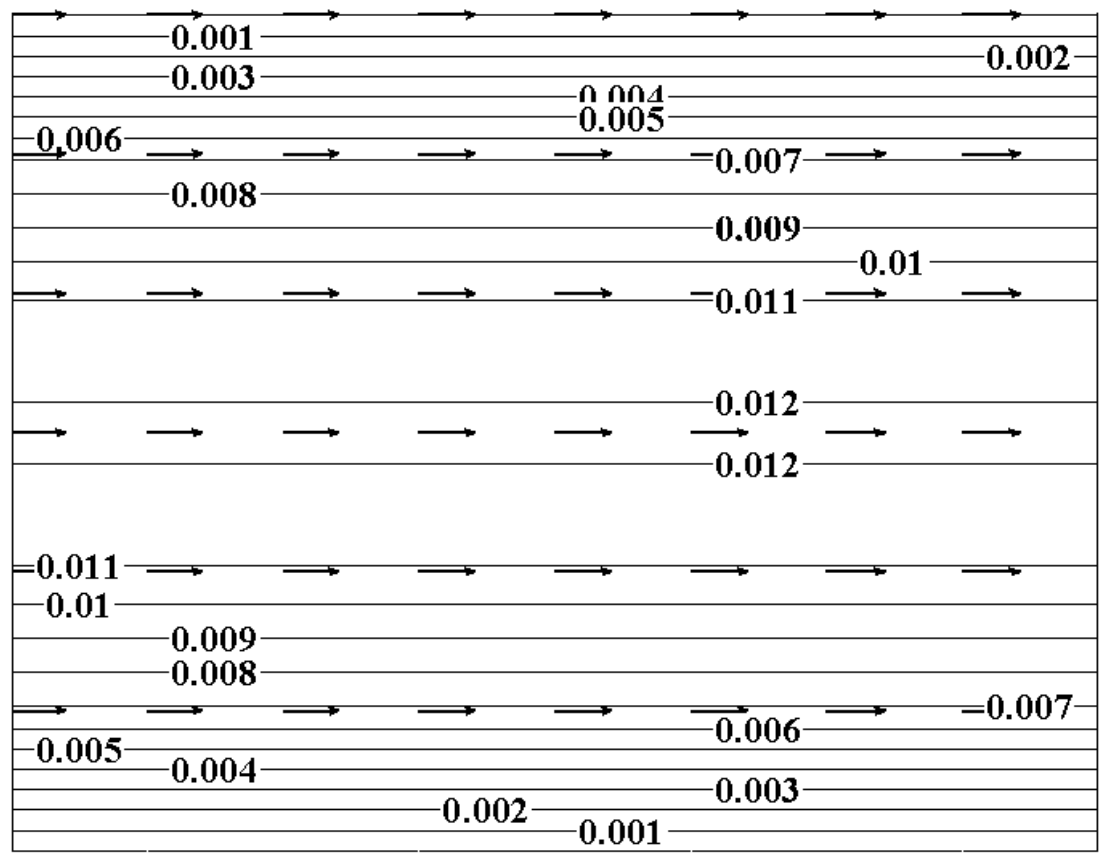

c) distribution of flow rate under entrance pressure

Fig.3 Distributions of the flow rate and pressure of fluids 
Table 1 Comparison of flow rate of outlet nodes under the superimposed effect of single boundary conditions and compound boundary conditions

\begin{tabular}{lllll}
\hline $\begin{array}{l}\text { Node } \\
\text { No. }\end{array}$ & $\begin{array}{l}\text { Flow rate under the } \\
\text { drag of walls }\end{array}$ & $\begin{array}{l}\text { Flow rate } \\
\text { under the } \\
\text { entrance } \\
\text { pressure }\end{array}$ & $\begin{array}{l}\text { Accumulated flow rate } \\
\text { at the outlet }\end{array}$ & $\begin{array}{l}\text { Flow rate under } \\
\text { the compound } \\
\text { effect at the outlet }\end{array}$ \\
\hline 3 & 0 & 0 & 0 & 0 \\
29 & 0.0033 & 0.0068 & 0.0101 & 0.0102 \\
8 & 0.0067 & 0.0109 & 0.0176 & 0.0176 \\
42 & 0.0100 & 0.0123 & 0.0223 & 0.0223 \\
13 & 0.0133 & 0.0109 & 0.0242 & 0.0243 \\
55 & 0.0167 & 0.0068 & 0.0235 & 0.0235 \\
18 & 0.0200 & 0 & 0.0200 & 0.0200 \\
\hline
\end{tabular}

\section{Results and Discussion}

Mainly aiming at two dimensional Newtonian rheological models with regular shapes, this work solved the flow rate and pressure of polymer fluids based on the FEM. Quadrilateral elements were used to discretize the grids in whole computing region. Then, according to the continuity of Navier-Stock equations, a weighted residual equation and a matrix element equation were established using an interpolation function and Jacobian matrix. Afterwards, these equations were combined to a general equation to further obtain the final flow rate and pressure of fluids. This method is also applicable to the flow of most fluids with regular shapes by generating corresponding computing regions and node information in the topology distribution of nodes in the program for the discretization of grids. The number of grids can be increased in the discretization of grids. However, as iterative computations are not needed in the solution of Newtonian fluids, the final precision of data obtained is slightly influenced.

\section{References}

[1] J N Reddy, D K Gartling. The Finite Element Method in Heat Transfer and Fluid dynamics. 2th ed., Boca Raton, CRC Press Inc, 2001.

[2] Akhtar W, Nazar M. Exact solutions for the rotational flow of generalized Maxwell fluids in a circular cylinder, J.Sci.Math.RoumanieTome, 2008, 51(99): 93-101.

[3] Fetecau C, Mahmood A, Corina Fetecau,et al. Some exact solurions for thehelical floe of a generalized Oldroyd-B fluid in a circular cylinder, J.Computers and mathematics with Applications, 2008, 56: 3096-3108.

[4] Ohta M, Nakamura T, Yoshida Y, et al. Lattice Boltzmann simulations of viscoplastic fluid flows through complex flow channels, J.Non-Newtonian Fluid Mechanics, 2011, 166(7): 404-412.

[5] Nejat A, Abdollahi V, Vahidkhah K. Lattice Boltzmann simulation of non-Newtonian flows past confined cylinders, J. Non-Newtonian Fluid Mechanics, 2011, 166(12): 689-697.

[6] Pei-xuan XU, Polymer rheology and its application. Chemical Industry Press, Bei Jing, 2003.

[7] Chao BI, Computational fluid mechanics finite element method and its programming. Machinery Industry Press, Bei Jing, 2013. 